\title{
XLVIII. Some ellipsoidal potentials, ÆEolotropic and isotropic
}

\section{R. Hargreaves M.A.}

To cite this article: R. Hargreaves M.A. (1906) XLVIII. Some ellipsoidal potentials, Eolotropic and isotropic, Philosophical Magazine Series 6, 11:64, 568-586, DOI: 10.1080/14786440609463472

To link to this article: http://dx.doi.org/10.1080/14786440609463472

曲 Published online: 16 Apr 2009.

Submit your article to this journal $[\pi$

ЏII Article views: 2

Q View related articles $\square$ 
(2) Different equations of state, all of which are fair approximations to the behaviour of a real gas, indicate very different values for these inversion temperatures.

(3) The sensitiveness of the positions of these points to change in the characteristic equation of the fluid makes a knowledge of their actual position, as determined experimentally, a very valuable means of discriminating between the relative validity of any proposed equations of state.

In conclusion attention may be drawn to the fact that all the results given in this paper are exact consequences of the equations of state to which they relate.

XIVIII. Some Ellipsoidal Potentials, Aolotropic and Isotropic. By R. HARGREAVES, M.A.**

TN a former paper on Æolotropic Potential, the potential 1 functions corresponding to normal distribution on a conducting ellipsoid and to uniform volume-distribution were treated. The functions are $\int_{\lambda}^{\infty} \frac{d \lambda}{\sqrt{J}}$ and $\int_{\lambda}^{\infty} \frac{\left(1-u_{\alpha}\right) d \lambda}{\sqrt{J}}$, where $\mathrm{J}$ is the cubic in $\lambda$ which replaces the isotropic form $\left(a^{2}+\lambda\right)\left(b^{2}+\lambda\right)\left(c^{2}+\lambda\right)$, and $u_{a}=1$ represents the xolian quadric which replaces a confocal. If the additional factor $\frac{\partial u_{a}}{\partial x}$ occurs under the integral sign a vector type is given, which was also considered in the second, but not in the first case.

It is proposed now to deal with the more general types

$$
\int_{\lambda}^{\infty} \frac{d \lambda}{\sqrt{ } \bar{J}}\left[u_{a}^{s}, \quad \text { or } u_{a}^{s}\left(1-u_{a}\right), \text { or }\left(1-u_{a}\right)^{s}\right]
$$

and with types in which the factor $\frac{\partial u_{\alpha}}{\partial x}$ is attached to the several forms. The second and third forms belong to volume distributions with densities depending on powers of $u_{a}$; the first has also a normal surface-density.

The energies attaching to these potentials take the simple form of numerical multiples of that of a conductor. They are obtained by the use of what (in the paper cited) were called wave-forms of the potential. Two theorems are required for the purpose: one to connect the wave-forms with

* Communicated by the Author. 
those written above, and the other to deal with moments and products of inertia of any even order for an ellipsoid. The evaluation of energy turns on a similarity in the forms of the two theorems, and they prove to be closely related.

A potential is then considered which is a linear function of members of the second type, namely,

$$
\int_{\lambda}^{\infty}\left(1-u_{\alpha}\right)\left(k_{1}+k_{2} u_{\alpha}+k_{3} u_{\alpha}^{2}+\ldots k_{n} u_{\alpha}^{n-1}\right) \frac{d \lambda}{\sqrt{J}} .
$$

The energy is a quadric in the $k$ 's, persymmetric in form; while the whole charge is a linear function of the $k$ 's. For any value of $n$ this potential may be determined so as to give a minimum of energy subject to constancy of total charge. The minima have simple values which decrease as $n$ increases, ranging from that for uniform volume-distribution $(n=1)$ to that for normal distribution on a conductor $(n=\infty)$. Thus the potentials form an interesting series of links connecting the two potentials commonly considered with reference to an ellipsoid; and each potential, with a distribution depending on $n$ constants, in some measure simulates that of a structure containing $n$ parts brought into relation by a minimum condition.

To give greater generality the work is written in æolotropic form; but most of the energies evaluated vary as $\int_{0}^{\infty} \frac{d \lambda}{\sqrt{ } \bar{J}}$, and the only difference between isotropic and rolotropic forms consists in the relation of the constants in the cubic $J$ to the axes of the ellipsoid.

$\S 1$. Of the three forms*

$$
\begin{aligned}
\psi_{s}=\frac{\rho}{4} \int_{\lambda}^{\infty} \frac{u_{\alpha}^{s} d \lambda}{\sqrt{J}}, \mathrm{U}_{s} & =\frac{\rho}{4} \int_{\lambda}^{\infty} \frac{u_{\alpha}^{s}\left(1-u_{\alpha}\right) d \lambda}{\sqrt{J}}, \\
\mathrm{~V}_{s} & =\frac{\rho}{4} \int_{\lambda}^{\infty} \frac{\left(1-u_{a}\right)^{s} d \lambda}{V^{\prime} \bar{J}}, . .
\end{aligned}
$$

the first will be taken as fundamental, results for $\mathrm{U}_{s}$ and $\mathrm{V}$. being directly deducible from those for $\psi_{s}$. Since $u_{\alpha}$ may be written $=1$ when it is not under the sign of integration, we have

$$
\frac{d \psi_{s}}{d x}=-\frac{\rho}{4 \sqrt{ } \bar{J}} \frac{d \lambda}{d x}+\frac{s \rho}{4} \int_{\lambda}^{\infty} u_{a}^{s-1} \frac{\partial u_{a}}{\partial x} \frac{d \lambda}{\sqrt{ } \bar{J}} .
$$

* The notation is that of the previous paper on 'Eolotropic Potential' in Phil. Mag. April 1905, quoted as $A$.

Phil. Mag. S. 6. Vol. 11. No. 64. April 1906. 2 P 
Thus the surface-discontinuity or density is the same for all the functions $\psi$ and is that of $\psi_{0}$; while potentials $\mathrm{U}_{s}$ and $\mathrm{V}_{s}$ containing differences of powers of $u_{a}$ have only a volume-density. A second differentiation gives

$$
\begin{aligned}
\frac{d^{2} \psi^{s}}{d x d y}= & -\frac{\rho}{4 \sqrt{J}}\left(\frac{d^{2} \lambda}{d i x d y}-\frac{\dot{J}}{2 J} \frac{d \lambda}{d x} \frac{d \lambda}{d y}\right)-\frac{s \rho}{4 \sqrt{J}} \frac{\partial u_{a}}{\partial x} \frac{d \lambda}{d y} \\
& +\frac{s \rho}{4} \int_{\lambda}^{\infty}\left\{2 \gamma^{\prime} u_{\alpha}^{s-1}+\overline{s-1} u_{\alpha}^{s-2} \frac{\partial u_{a}}{\partial x} \frac{\partial u_{a}}{\partial y}\right\} \frac{d \lambda}{\sqrt{J}} .
\end{aligned}
$$

When $\nabla_{e}^{2} \psi_{s}$ is formed, the sum of terms such as those in the first bracket vanishes, and the total outside the sign of integration is $-s \rho / \sqrt{\mathrm{J}}$. Under the sign of integration the brackst gives

or

$$
2 u_{a}^{s-1} \Sigma\left(p \boldsymbol{\alpha}+2 p^{\prime} \alpha^{\prime}\right)-4(s-1) u_{a}^{s-2} i_{a},
$$

$$
2 u_{\alpha}^{s-1} \dot{\mathrm{J}} / \mathrm{J}-4(s-1) u_{\alpha}^{s-2} \dot{u}_{\alpha} \text { quoting } \mathbb{E} .(76) \text { and (86). }
$$

Hence the integral

$$
=-s \rho \int_{\lambda}^{\infty} \frac{\partial}{\partial \lambda}\left(\frac{u_{\alpha}^{s-1}}{\sqrt{ } / \mathrm{J}}\right) d \lambda=s \rho u_{\alpha}^{s-1} / \sqrt{\mathrm{J}}
$$

Externally the lower limit is variable, and the two terms are cancelled in virtue of $u_{a}=1$; thus $\nabla_{e}^{2} \psi_{s}=0$. Internally the lower limit is constant and $=0$, and there are no terms outside the integral sign; since $\lambda=0$ makes $J=1, u_{\alpha}=u_{a}$, the integral is $s \rho u_{a}^{s-1}$, that is $\nabla_{e}^{2} \psi_{s}=s \rho u_{a}^{s-1}$ and the volume-density is - $s \rho u_{a}^{s-1}$. The surface-density $\sigma$

$$
\begin{aligned}
= & -\left[l\left(p \frac{d \psi_{0}}{d x}+r^{\prime} \frac{d \psi_{0}}{d y}+q^{\prime} \frac{d \psi_{0}}{d z}\right)+m\left(r^{\prime} \frac{d \psi_{0}}{d x}+q^{\frac{d \psi_{0}}{d y}}+p^{\prime} \frac{d \psi_{0}}{d z}\right)\right. \\
& \left.+n\left(q^{\prime} \frac{d \psi_{0}}{d x}+p^{\prime} \frac{d \psi_{0}}{d y}+r \frac{d \psi_{0}}{d z}\right)\right] \\
= & \frac{\rho}{4}\left[l\left(p \frac{d \lambda}{d x}+r^{\prime} \frac{d \lambda}{d y}+q^{\prime} \frac{d \lambda}{d z}\right)+\ldots\right] \\
= & \frac{\rho \sigma}{8}\left[\frac{\partial u_{a}}{\partial x}\left(p \frac{d \lambda}{d x}+r^{\prime} \frac{d \lambda}{d y}+q^{\prime} \frac{d \lambda}{d z}\right)+\ldots\right]=\frac{\rho \varpi}{2},
\end{aligned}
$$

w being a perpendicular from the centre on a tangent plane and $l m n$ direction-cosines of a normal.

$$
\text { Since } \frac{d \psi_{s}}{d x}=\frac{d \psi_{0}}{d x}+\frac{\partial \psi_{s}}{\partial x} \text {, and } \frac{d \psi_{s}}{d x} \text { and } \frac{d \psi_{0}}{d x} \text { are external }
$$


potentials, so also is $\frac{\partial \psi_{s}}{\partial x}$. It is more convenient to use an independent notation for the vector type, and we take

$$
\chi_{s}=\int_{\lambda}^{\infty} \frac{u_{\alpha}^{s}\left(\alpha x+\gamma^{\prime} y+\beta^{\prime} z\right) d \lambda}{\sqrt{ } \mathcal{J}} .
$$

Since

$$
\begin{aligned}
& \frac{d \chi_{s}}{d x}=-\frac{\rho}{4 \sqrt{ } \mathbf{J}}\left(\alpha x+\gamma^{\prime} y+\beta^{\prime} z\right) \frac{d \lambda}{d x} \\
& +\frac{\rho}{4} \int_{\lambda}^{\infty}\left[\alpha u_{a}^{s}+s u_{\alpha}^{s-1}\left(a x+\gamma^{\prime} y+\beta^{\prime} z\right) \frac{\partial u_{a}}{\partial x}\right] \frac{d \lambda}{\sqrt{\bar{J}}},
\end{aligned}
$$

the surface-discontinuity is the same as for $\chi_{0}$, and the surface-density is $\frac{\rho \sigma}{2}\left(a x+c^{\prime} y+b^{\prime} z\right)$. The volume-density is $-s p\left(a x+c^{\prime} y+b^{\prime} z\right) u_{a}^{s-1}$ as appears from the connexion with $\psi_{s+1}$; or by work following closely the lines of $\mathrm{E}$. p. 442 we show that

$$
\begin{aligned}
& \nabla_{e}^{2} \chi_{s}=-s \rho\left(\alpha x+\gamma^{\prime} y+\beta^{\prime} z\right) / \sqrt{\mathrm{J}} \\
& -s \rho \int_{\lambda}^{\infty} \frac{\partial}{\partial \lambda}\left\{\frac{u_{a}^{s-1}\left(\alpha x+\gamma^{\prime} y+\beta^{\prime} z\right)}{\sqrt{ } \bar{J}}\right\} d \lambda \\
& =-s \rho\left(\alpha x+\gamma^{\prime} y+\beta^{\prime} z\right) / \sqrt{ } \bar{J}+s \rho u_{\alpha}^{s-1}\left(\alpha x+\gamma^{\prime} y+\beta^{\prime} z\right) / \sqrt{ } \bar{J},
\end{aligned}
$$

from which

$$
\nabla_{e}^{2} \chi_{s}=0 \text { externally and }=s \rho\left(a x+c^{\prime} y+b^{\prime} z\right) u_{a}^{s-1} \text { internally. }
$$

$\S 2$. We proceed to the formulæ by which the above are transferred to wave-forms. In the latter it is more convenient to work with $\mu=\lambda \Delta_{a}$ and $\Delta(\mu)=\Delta_{a}^{2} J(\lambda)$, identity in final results following from $d \mu / \sqrt{\Delta(\mu)}=d \lambda / \sqrt{J}$. The fundamental formula for transference is

$$
\frac{\Delta_{a}^{s}}{2 \pi} \int \frac{\left(l_{x}+m y+n z\right)^{2 s} d \omega}{u_{p}\left(u_{\mathrm{A}}+\mu u_{p}\right)^{s+1 / 2}}=\int_{\mu}^{\infty} \frac{u_{\alpha}^{s} d \mu}{\sqrt{\Delta(\mu)}}=\int_{\lambda}^{\infty} \frac{u_{\alpha}^{s} d \lambda}{\sqrt{ } \bar{J}}=\psi_{s},
$$

and a particular case is

$$
\frac{\Delta_{a}^{s}}{2 \pi} \int \frac{(l x+m y+n z)^{2 s} d \omega}{u_{p} u_{\mathrm{A}}^{s+1 / 2}}=\int_{0}^{\infty} \frac{u_{\alpha}^{s} d \lambda}{\sqrt{\mathrm{J}}} ; .
$$

the general case applies to external potentials, the particular to internal potentials, the latter specially required for energy. Now (3) is an immediate integral, with regard to $\mu$, of

$$
\begin{aligned}
\frac{1}{4 \pi} \int \frac{(l x+m y+n z)^{2 s} d \omega}{\left(u_{\Delta}+\mu u_{p}\right)^{s+3 / 2}} & =\frac{1}{2 s+1}\left(\frac{u}{\Delta_{a}}\right)^{s} \frac{1}{\sqrt{\overline{\Delta(\mu)}}} \\
& =\frac{u_{A(\mu)}^{s}}{(2 s+1)\{\Delta(\mu)\}^{s+1 / 2}},
\end{aligned}
$$


the particular case with $\mu=0$ being

$$
\frac{1}{4 \pi} \int \frac{(l a+m y+n z)^{2 s} d \omega}{u_{\mathrm{A}}^{s+3 / 2}}=\frac{u_{a}^{s}}{(2 s+1) \Delta_{a}^{s+1}} \geqslant .
$$

$u_{a}$ and $u_{a}$ on the right hand have ( $x y z$ ) for variables, $u_{\mathrm{A}}$ and $u_{p}$ on the left have $(\ln n)$. We shall deduce (4) from

$$
\frac{1}{4 \pi} \int \frac{d \omega}{\left(u_{A}+\mu u_{p}\right)^{3 / 2}}=\frac{1}{\sqrt{\Delta(\mu)}}, \quad \cdots
$$

an integral obtained indirectly in the previous paper, and first give an independent proof of (5). The volume integral

$$
\iiint d x d y d z / \sqrt{\frac{x^{2}}{\alpha^{2}}+\frac{y^{2}}{\beta^{2}}+\frac{z^{2}}{\gamma^{2}}}=2 \pi \alpha \beta \gamma
$$

where the range of integration is the volume contained by the ellipsoid $\frac{x^{2}}{\alpha^{2}}+\frac{y^{2}}{\beta^{2}}+\frac{z^{2}}{\gamma^{2}}=1$, is well known. Put $(x, y, z)=r(l, m, n)$, and use a polar element of volume $r^{2} d r d \omega$, where $d \omega$ is an element of area on a unit sphere; then integrate with regard to $r$, the boundary value being $1=\mathrm{R}^{2}\left(\frac{l^{2}}{a^{2}}+\frac{m^{2}}{\beta^{2}}+\frac{n^{2}}{\gamma^{2}}\right) . \quad$ The result is

$$
\frac{1}{4 \pi} \int d \omega /\left(\frac{l^{2}}{\alpha^{2}}+\frac{m^{2}}{\beta^{2}}+\frac{n^{2}}{\gamma^{2}}\right)^{3 / 2}=\alpha \beta \gamma,
$$

or

$$
\frac{1}{4 \pi} \int d \omega /\left(a l^{2}+b m^{2}+c n^{2}\right)^{3 / 2}=\frac{1}{\sqrt{a b c}} .
$$

If the ellipsoid is referred to general axes, then $u_{\alpha}(l, m, n)$ takes the place of $a l^{2}+b m^{2}+c n^{2}$, and $\alpha b c$ must be replaced by $\Delta_{a}$. If again for $a$ we write $\mathrm{A}+\mu p$, for $a^{\prime}, \mathrm{A}^{\prime}+\mu p^{\prime}$, then $\Delta_{a}$ is replaced by $\Delta(\mu)$, a determinant with the elements $\mathrm{A}+\mu p, \ldots$ and this is (5).

The step to (4) is made by using an operator

$$
\Sigma\left(x^{2} \frac{d}{d \mathrm{~A}}+y=\frac{d}{d \mathrm{~A}^{\prime}}\right) \text { say } 0 .
$$

Since

$$
\frac{d}{d \mathrm{~A}} u_{\mathrm{A}}(l, m, n)=l^{2}, \frac{d}{d \mathrm{~A}^{\prime}} u_{\mathrm{A}}=2 m n, \ldots
$$

we have

$$
\text { ○. } u_{\mathbf{A}}(l, m, n)=(l x+m y+n z)^{2} \text {. }
$$


And since

$$
\begin{aligned}
& \frac{d}{d \mathrm{~A}} \Delta(\mu)=\mathrm{A}(\mu)=\frac{\alpha \Delta(\mu)}{\Delta_{a}}, \text { and } \frac{d}{d \mathrm{~A}^{\prime}} \Delta(\mu)=2 \mathrm{~A}^{\prime}(\mu)=\frac{2 \alpha^{\prime} \Delta(\mu)}{\Delta_{a}}, \ldots \\
& \text { we have } \quad 0 . \Delta(\mu)=u_{\mathrm{A}(\mu)}(x, y, z)=\frac{u_{a} \Delta(\mu)}{\Delta_{a}} .
\end{aligned}
$$

Also $u_{\mathrm{A}(\mu)}$ written at length is

$$
\begin{gathered}
\Sigma x^{2}\left\{\left(\mathrm{BC}-\mathrm{A}^{\prime 2}\right)+\mu\left(q \mathrm{C}+r \mathrm{~B}-2 p^{\prime} \mathrm{A}\right)+\mu^{2} \mathrm{P}\right\} \\
+2 \Sigma y=\left\{\mathrm{B}^{\prime} \mathrm{C}^{\prime}-\mathrm{AA}^{\prime}+\mu\left(q^{\prime} \mathrm{C}^{\prime}+r^{\prime} \mathrm{B}^{\prime}-p \mathrm{~A}^{\prime}-p^{\prime} \mathrm{A}\right)+\mu^{2} \mathrm{P}^{\prime}\right\}
\end{gathered}
$$

and therefore

$$
\begin{aligned}
& \frac{d}{d \mathrm{~A}^{2}} u_{\mathrm{A}(\mu)}=(\mathrm{C}+\mu r) y^{2}+(\mathrm{B}+\mu q) z^{2}-2\left(\mathrm{~A}^{\prime}+\mu p^{\prime}\right) y z, \\
& \frac{1}{2} \frac{d}{d \mathrm{~A}^{\prime}} u_{\mathrm{A}(\mu)}=\left(\mathrm{C}^{\prime}+\mu r^{\prime}\right) z x+\left(\mathrm{B}^{\prime}+\mu q^{\prime}\right) y x-(\mathrm{A}+\mu p) y z-\left(\mathrm{A}^{\prime}+\mu p^{\prime}\right) x^{2} .
\end{aligned}
$$

When the whole operator is used all terms are cancelled, and we have the three results

$$
\begin{aligned}
& \text { O. } u_{\mathbf{A}}(l, m, n)=(l x+m y+n z)^{2}, O \cdot \Delta(\mu)=u_{\mathbf{A}(\mu)}(x, y, z)=\frac{u_{a} \Delta(\mu)}{\Delta_{a}}, \\
& \text { and } \quad \text { O. } u_{\mathbf{A}(\mu)}(x, y, z)=0 .
\end{aligned}
$$

Hence the operation applied to the first and third members of (4) yields a repetition of (4) with $s+1$ for $s$; and (4) follows by repeated use of the operator starting from (5).

The formula of transference when $\alpha x+\gamma^{\prime} y+\beta^{\prime} z$ appears under the integral sign is got from (4) by use of the operator

for which

$$
\mathrm{O}_{x}^{\prime}=x \frac{d}{d \mathrm{~A}}+\frac{y}{2} \frac{d}{d \mathrm{C}^{\prime}}+\frac{z}{2} \frac{d}{d \mathrm{~B}^{\prime}},
$$

$$
\left.\begin{array}{cl}
\mathrm{O}_{x}{ }^{\prime} \cdot u_{\mathrm{A}}=l(l x+m y+n z), & \mathrm{O}_{x}{ }^{\prime} \cdot \Delta(\mu)=\frac{\Delta(\mu)}{\Delta_{a}}\left(\alpha x+\gamma^{\prime} y+\beta^{\prime} z\right), \\
& \mathrm{O}_{x}{ }^{\prime} \cdot u_{\Delta(\mu)}=0 .
\end{array}\right\}
$$

This gives

$$
\frac{1}{4 \pi} \int \frac{l(l x+m y+n z)^{2 s-1} d \omega}{\left(u_{\Delta}+\mu u_{p}\right)^{s+3 / 2}}=\frac{u_{a}^{s-1}\left(\alpha x+\gamma^{\prime} y+\beta^{\prime} z\right)}{(2 s+1) \Delta_{a}^{s} \sqrt{\Delta(\mu)}} .
$$

Integrate with regard to $\mu$ from $\mu$ to $\infty$ and raise $s$ by 1 , then

$$
\frac{\Delta_{a}^{s+1}}{2 \pi} \int \frac{l(l x+m y+n z)^{2 s+1} d \omega}{u_{p}\left(u_{\mathrm{A}}+\mu u_{p}\right)^{s+3 / 2}}=\int_{\mu}^{\infty} \frac{u_{a}^{s}\left(\alpha x+\gamma^{\prime} y+\beta^{\prime} z\right) d \mu}{\sqrt{\Delta(\mu)}},
$$


and in particular

$$
\frac{\Delta_{a}^{s+1}}{2 \pi} \int \frac{l(l x+m y+n z)^{2 s+1} d \omega}{u_{p} u_{\Lambda}^{s+3 / 2}}=\int_{0}^{\infty} \frac{u_{a}^{s}\left(\alpha x+\gamma^{\prime} y+\beta^{\prime} z\right) d \mu}{\sqrt{\Delta(\mu)}}(10 b)
$$

$\$ 3$. The fundamental integral for moments of inertia in an ellipsoid is

$$
\frac{1}{\tau_{0}} \int(l x+m y+n z)^{2 s} d \tau=\frac{3}{(2 s+1)(2 s+3)}\left\{\frac{{ }^{u_{A}}(l, m, n)}{\Delta_{a}}\right\}^{s}
$$

The coefficients of like power arrangements in $l m n$ are equal on the two sides, so that all moments and products of inertia of degree $2 s$ are comprised in the formula. A way of expressing the results for individual terms, which also applies to (4), will be given later ; but for our main purpose the form (11) and some collateral results are required. In immediate connexion with (11) are

$$
\frac{1}{\tau_{0}} \int(l x+m y+n z)^{2 s} u_{a}^{s^{\prime}} d \tau=\frac{3}{(2 s+1)} \frac{3}{\left(2 s+2 s^{\prime}+3\right)}\left(\frac{u_{\mathrm{A}}}{\Delta_{a}}\right)^{s}
$$

and the surface integral

$$
\frac{1}{\tau_{0}} \int(l x+m y+n z)^{2 s} \varpi d \mathrm{~S}=\frac{3}{2 s+1}\left(\frac{u_{\mathrm{A}}}{\Delta_{a}}\right)^{s}, .
$$

where $\sigma$ is a perpendicular from the centre on a tangent plane. Write $x, y, z=r(\lambda, \mu, \nu)$, use a polar element of volume $r^{2} d r d \omega$ and integrate to the surface value

For (11)

$$
1=\mathrm{R}^{2} u_{a}(\lambda, \mu, \nu) .
$$

$$
\begin{aligned}
\int(l x+m y+n z)^{2 s} d \tau & =\iint r^{2 s+2}(l \lambda+m \mu+n \nu)^{2 s} d r d \omega \\
& =\frac{1}{2 s+3} \int \mathrm{R}^{2 s+3}(l \lambda+m \mu+n \nu)^{2 s} d \omega
\end{aligned}
$$

for $(11 b) \int(l x+m y+n z)^{s s} u_{a}^{s^{\prime}} d \tau$

$$
\begin{aligned}
& =\frac{1}{2 s+2 s^{\prime}+3} \int \mathrm{R}^{2 s+2 s^{\prime} \pm 3}(l \lambda+m \mu+n \nu)^{2 s} u_{a}^{s^{\prime}}(\lambda, \mu, \nu) d \omega \\
& =\frac{1}{2 s+2 s^{\prime}+3} \int \mathrm{R}^{2 s+3}(l \lambda+m \mu+n \nu)^{2 s} d \omega
\end{aligned}
$$

and for $(11 c)$

$$
\int(l x+m y+n z)^{2 s} \varpi d \mathrm{~S}=\int \mathrm{R}^{2 s+3}(l \lambda+m \mu+n v)^{2 s} d \omega,
$$

since $\varpi d \mathrm{~S}=\mathrm{R}^{3} d \omega$. 
Thus the three forms all depend on one angular integral which, in view of the value of $\mathrm{R}$, and $\tau_{0}=4 \pi / 3 \sqrt{\Delta_{a}}$, is

$$
\frac{1}{4 \pi} \int \frac{(l \lambda+m \mu+n v)^{2 s} d \omega}{\left\{u_{a}(\lambda, \mu, \nu)\right\}^{s+3 / 2}}=\frac{\left\{u_{\Lambda}(l, m, n)\right\}^{s}}{(2 s+1) \Delta_{a}^{s+1 / 2}} .
$$

This integral is a variant of $(4 b)$ got by writing $a$ for $\mathrm{A}$.

A modification of (11) by a factor $a x+c^{\prime} y+b^{\prime} z$ is required for the $\chi$ functions. Operate with $a \frac{d}{d l}+c^{\prime} \frac{d}{d m}+b^{\prime} \frac{d}{d n}$ on (11), and raise $s$ by 1 , thus

$$
\begin{aligned}
\frac{1}{\tau_{0}} \int\left(a x+c^{\prime} y+b^{\prime} z\right)(l x+m y+n z)^{2 s+1} d \tau & \\
& =\frac{3 l}{(2 s+3)(2 s+5)}\left(\frac{u_{\mathrm{A}}}{\Delta_{a}}\right)^{s} ; .
\end{aligned}
$$

then

$$
\begin{gathered}
\frac{1}{\tau_{\mathrm{o}}} \int\left(a x+c^{\prime} y+b^{\prime} z\right)(l x+m y+n z)^{2 s+1} u_{a}^{s^{\prime}} d \tau \\
=\frac{3 l}{(2 s+3)\left(2 s+2 s^{\prime}+5\right)}\left(\frac{u_{\mathrm{A}}}{\Delta_{a}}\right)^{s},
\end{gathered}
$$

and

$$
\begin{array}{r}
\frac{1}{\tau_{\mathrm{o}}} \int\left(a x+c^{\prime} y+b^{\prime} z\right)(l x+m y+n z)^{2 s+1} \varpi d \mathrm{~S} \\
=\frac{3 l}{2 s+3}\left(\frac{u_{\mathrm{A}}}{\Delta_{a}}\right), \ldots . . .
\end{array}
$$

are connected with (12) in the manner shown above for $(11 b)$ and $(11 c)$.

$\S 4$. The evaluation of energy now follows by combining the typical integrals, thus :-

$$
\begin{aligned}
\frac{1}{\tau_{0}} \int u_{a}^{s^{\prime}} d \tau \int_{0}^{\infty} \frac{u_{a}^{s} d \lambda}{\sqrt{J}} & =\frac{1}{\tau_{0}} \int u_{a}^{s^{\prime}} d \tau \int \frac{\Delta_{a}^{s}(l x+m y+n z)^{2 s} d \omega}{2 \pi u_{p} u_{\mathrm{A}}^{s+1 / 2}} \text { by }(3 b) \\
& =\frac{3}{(2 s+1)\left(2 s+2 s^{\prime}+3\right)} \int \frac{d \omega}{2 \pi u_{p} u_{\mathbf{A}}^{1 / 2}} \text { by }(11 b) \\
& =\frac{3 \phi_{0}}{(2 s+1)\left(2 s+2 s^{\prime}+3\right)} \cdot \text {. . . . (13) }
\end{aligned}
$$

using $\phi_{0}$ as in E. (135) for

$$
\int_{0}^{\infty} \frac{d \lambda}{\sqrt{J}}=\int_{0}^{\infty} \frac{d \mu}{\sqrt{\Delta(\mu)}}=\int \frac{d \omega}{2 \pi u_{p} u_{\mathrm{A}}^{1 / 2}}
$$

We may also write (13) as

$$
\int u_{a}^{s^{\prime}} \psi_{s} d \tau=\frac{3 \rho \tau_{0} \phi_{0}}{4(2 s+1)\left(2 s+2 s^{\prime}+3\right)}, .
$$


and the surface integral is

$$
\int \psi_{s} \varpi d \mathrm{~S}=\frac{3 \rho \tau_{0} \phi_{0}}{4(2 s+1)} \ldots \ldots
$$

Now the potential $\psi_{s^{\prime}}$ has a surface-density $\rho \varpi / 2$, and a volume-density $-s^{\prime} \rho u_{a}^{s-i}$. The term in energy due to the interaction of $\psi_{s}$ and $\psi_{s}$ is therefore

$$
\begin{aligned}
\mathrm{E}\left(s, s^{\prime}\right) & =\frac{1}{2} \int \rho \varpi \psi d \mathrm{~S}-\int s^{\prime} \rho u_{a}^{s^{\prime}-1} \psi_{s} d \tau \\
& =\frac{3 \rho^{2} \tau_{0}}{8}\left(\frac{1}{2 s+1}-\frac{2 s^{\prime}}{2 s+1-\overline{2 s+2 s^{\prime}+1}}\right)=\frac{3 \rho^{2} \tau_{0} \phi_{o}}{8\left(2 s+2 s^{\prime}+1\right)}
\end{aligned}
$$

The energy due to $\psi_{s}$ alone is $3 \rho^{2} \tau_{0} \phi_{0} / 16(4 s+1)$, i.e. we write $s^{\prime}=s$ and halve the result. The energy belonging to $k_{s} \psi_{s}+k_{s^{\prime}} \psi_{s^{\prime}}$ is

$$
\frac{3 \rho^{2} \tau_{0} \phi_{0}}{16}\left[\frac{k_{s}^{2}}{4 s+1}+\frac{2 k_{s} k_{s^{\prime}}}{2 s+2 s^{\prime}+1}+\frac{k_{s^{\prime}}^{2}}{4 s^{\prime}+1}\right],
$$

but it is clearly sufficient to give as in (14) a single composite term. The total charge attaching to $\psi_{s}$ is

$$
\frac{1}{2} \int \rho \varpi d S-\int s \rho u_{a}^{s-1} d \tau=\frac{3 \rho \tau_{o}}{2}-\frac{3 s \rho \tau_{0}}{2 s+1}=\frac{3 \rho \tau_{0}}{2(2 s+1)} .
$$

For normal distribution on a conductor $s=0, e=3 \rho \tau_{0} / 2$, and the energy is $e^{2} \phi_{0} / 12 \tau_{0}$.

From (14) may be derived the composite term in the energy of $k_{s} U_{s}+k_{s^{\prime}} \dot{U}_{s^{\prime}}$, or of $k_{s}\left(\psi_{s}-\psi_{s+1}\right)+k_{s^{\prime}}\left(\psi_{s^{\prime}}-\psi_{s^{\prime}+1}\right)$. For this term we are concerned with the products

$$
\rho_{s^{\prime}} \psi_{s}-\rho_{s^{\prime}+1} \psi_{s}-\rho_{s^{\prime}} \psi_{s+1}+\rho_{s^{\prime}+1} \psi_{s+1},
$$

and the application of (14) yields

$$
\begin{array}{r}
\frac{3 \rho^{2} \tau_{0} \phi_{0}}{8}\left[\frac{1}{2 s+2 s^{\prime}+1}-\frac{2}{2 s+2 s^{\prime}+3}+\frac{1}{2 s+2 s^{\prime}+5}\right] \\
=\frac{3 \rho^{2} \phi_{0}}{2 s+2 s^{\prime}+1}-\overline{2 s+2 s^{\prime}+3} \overline{2 s+2 s^{\prime}+5}
\end{array}
$$

which multiplied by $k_{s} k_{s}$, is the composite term required.

To deal with the function $V_{s}$ or $\frac{\rho}{4} \int \frac{d \lambda}{\sqrt{J}}\left(1-u_{\alpha}\right)^{s}$, the two summations for the indices of $\rho$ and $\psi$ may be taken 
Potentials, Aiolotropic and Isotropic.

separately. The first is

and the second is

$$
\begin{aligned}
& \underset{8}{3 \rho^{2} \tau_{0} \phi_{0}}\left[1-\frac{s^{\prime} \mathrm{C}_{1}}{3}+\frac{s^{\prime} \mathrm{C}_{2}}{5}-\ldots \frac{\overline{-1} !^{s \prime}}{2 s^{\prime}+1}\right] \\
& =\frac{3 \rho^{2} \tau_{0} \phi_{0}}{8} \cdot \frac{2.4 \ldots 2 s^{\prime}}{3.5 \ldots \overline{2} s^{\prime}+3} ;
\end{aligned}
$$

$$
\begin{array}{r}
\frac{3 \rho^{2} \tau_{0} \phi_{0}}{8} \times 2.4 \ldots 2 s^{\prime}\left[\frac{1}{1.3 \ldots \overline{2 s^{\prime}+1}}-\frac{s \mathrm{C}_{1}}{3.5 \ldots 2 s^{\prime}+3}\right. \\
\left.+\ldots \frac{-1}{2 s+1 . \overline{2 s+3} \ldots \overline{2 s+2 s^{\prime}+1}}\right] \\
=\frac{3 \rho^{2} \tau_{\mathrm{o}} \phi_{\mathrm{o}}}{8} \cdot \frac{2.4 \ldots \overline{2 s+2 s^{\prime}}}{3.5 \ldots \overline{2 s+\overline{2 s^{\prime}+1}} ; \quad . \quad(16)}
\end{array}
$$

and this quantity multiplied by $k_{s} k$ is the composite term in the energy of $k_{s} \mathrm{~V}_{s}+k_{s^{\prime}} \mathrm{V}_{s^{\prime}}$. The summations for (16) are made by a series of steps in which the combination formula $\mathrm{C}=\underset{s-1}{\mathrm{C}}+{ }_{s-1}^{\mathrm{C}}$ is used, and the difference of adjoining terms taken. The densities aitaching to $\mathrm{U}_{s}$ and $\mathrm{V}_{s}$ are respectively $\rho\left(\overline{s+1} u_{a}^{s}-s u_{a}^{s-1}\right)$ and $s \rho\left(1-u_{a}\right)^{s-1}$, and the corresponding total charges $\frac{3 \rho \tau_{0}}{2 s+12 s+3}$ and $\frac{3 \rho \tau_{0}}{2} \cdot \frac{2.4 \ldots 2 s}{3.5 \ldots 2 s+1}$; they follow from those of $\psi_{s}$, or may be got by independent work. The energy formula for $U_{s}$ may be got directly, viz. for the composite term

$$
\begin{aligned}
& \mathrm{E}\left(s, s^{\prime}\right)=\int \rho_{s^{\prime}} \mathrm{U}_{s} d \tau \\
& =\int \rho\left(\overline{s^{\prime}+1} u_{a}^{s^{\prime}}-s^{\prime} u_{a}^{s^{\prime}-1}\right) d \tau \cdot \frac{\rho}{4} \int_{0}^{\infty} \frac{u_{a}^{a}\left(1-u_{a}\right) d \lambda}{\sqrt{J}} \text {, which by }(13) \\
& =\frac{3 \rho^{2} \tau_{0} \phi_{0}}{4}\left[\left(s^{\prime}+1\right)\left\{\frac{1}{\overline{2 s+12 s+2 s^{\prime}+3}}-\frac{1}{2 s+3} \overline{2 s+2 s^{\prime}+5}\right\}\right. \\
& \left.\quad-s^{\prime}\left\{\frac{1}{\overline{2 s+1}}-\frac{1}{2 s+2 s^{\prime}+1}-\frac{1}{2 s+3} \frac{2 s+2 s^{\prime}+3}{2 s}\right\}\right] \\
& =3 \rho^{2} \tau_{0} \phi_{0} /\left(2 s+2 s^{\prime}+1\right)\left(2 s+2 s^{\prime}+3\right)\left(2 s+2 s^{\prime}+5\right),
\end{aligned}
$$

in agreement with (15). Moreover, the energy of $\psi_{s}$ may be calculated from that of $U_{s}$ by treating $\psi_{s}$ as $\mathrm{U}_{s}+\mathrm{U}_{s+1}+\ldots$ ad inf., and making a double summation of the formula just obtained. Either $\mathrm{U}_{s}$ or $\mathrm{V}_{s}$ for $s=0$ gives the case of uniform volume distribution $e=\rho \tau_{0}$, and energy $=e^{2} \phi_{0} / 10 \tau_{0}$. 
$\S 5$. We now give corresponding results for $\chi_{s}$. Using the integrals, $(10 b)$ for the first step, and $(12 b)$ for the second,

$$
\begin{aligned}
& \frac{1}{\tau_{0}} \int u_{a}^{s^{\prime}}\left(\alpha x+c^{\prime} y+b^{\prime} z\right) d \tau \int_{0}^{\infty} \frac{u_{a}^{s}\left(\alpha x+\gamma^{\prime} y+\beta^{\prime} z\right) d \lambda}{\sqrt{ } \overline{\mathrm{J}}} \\
& =\frac{1}{\tau_{0}} \int u_{a}^{s^{\prime}}\left(a x+c^{\prime} y+b^{\prime} z\right) d \tau \int \frac{\Delta_{a}^{s+1} l(l x+m y+n z)^{2 s+1} d \omega}{2 \pi u_{p} u_{\mathrm{A}}^{s+3 / 2}} \\
& =\frac{3}{(2 s+3)\left(2 s+2 s^{\prime}+5\right)} \int \frac{\Delta_{a} l^{2} d \omega}{2 \pi u_{p} u_{\mathrm{A}}^{3 / 2}} \\
& =\frac{3 \mathrm{~L}_{0}}{(2 s+3)\left(2 s+2 s^{\prime}+5\right)}
\end{aligned}
$$

where. $L_{0}$ as in $\mathbb{E}$. (135) stands for

$$
\int_{0}^{\infty} \frac{\alpha d \lambda}{\sqrt{ } \mathbf{J}}=\int \frac{\Delta_{a} l^{2} d \omega}{2 \pi u_{p} u_{\mathbf{A}}^{3 / 2}}
$$

This and the corresponding surface integral may be written

$$
\int u_{a}^{s^{\prime}}\left(a x+c^{\prime} y+b^{\prime} z\right) \chi_{s} d \tau=\frac{3 \rho \tau_{0} \mathrm{~L}_{\mathrm{o}}}{4(2 s+3)\left(2 s+2 s^{\prime}+\overline{5}\right)} .
$$

and

$$
\int\left(a x+c^{\prime} y+b^{\prime} z\right) \chi_{s} \varpi d \mathrm{~S}=\frac{3 \rho \tau_{0} \mathrm{~L}_{0}}{4(2 s+3)} .
$$

The surface-density due to $\chi_{s^{\prime}}$ is $\frac{\rho \sigma}{2}\left(a x+c^{\prime} y+b^{\prime} z\right)$, and the volume-density $-s^{\prime} \rho u_{a}^{s^{\prime}-1}\left(a x+c^{\prime} y+b^{\prime} z\right)$; thus applying (17 $b$ and $c$ ) the composite term in energy attaching to indices $s$ and $s^{\prime}$ is

$$
\mathrm{E}\left(s, s^{\prime}\right)=\frac{3 \rho^{2} \tau_{0} \mathrm{~L}_{0}}{8(2 s+3)}\left[1-\frac{2 s^{\prime}}{2 s+2 s^{\prime}+3}\right]=\frac{3 \rho^{2} \tau_{0} \mathrm{~L}_{0}}{8\left(2 s+2 s^{\prime}+3\right)} .
$$

If functions with $\gamma^{\prime} x+\beta y+\alpha^{\prime} z$ and $\beta^{\prime} x+\alpha^{\prime} y+\gamma z$ are also under consideration, the three functions may be distinguished as $\chi_{s}(x), \chi_{s}(y), \chi_{s}(z)$. In the composite term for $\chi_{s}(y)$ and $\chi_{s^{\prime}}(y), \mathbf{M}_{0}$ appears for $\mathrm{L}_{0}$ above, but if a composite term for $\chi_{s}(x)$ and $\chi_{s^{\prime}}(y)$ is in question $\mathrm{N}_{0}^{\prime}$ appears, viz.,

$$
\mathrm{N}_{0}^{\prime}=\int \frac{\Delta_{a} l m d \omega}{2 \pi u_{p} u_{\mathrm{\Lambda}}^{3 / 2}}=\int_{0}^{\infty} \frac{\gamma^{\prime} d \lambda}{\sqrt{\mathrm{J}}} .
$$

For the density of $\chi_{s^{\prime}}(y)$ has the factor $c^{\prime} x+\dot{b} y+a^{\prime} z$; and if this is taken with $\chi_{s}(x)$ a factor $m$ is introduced in place of $l$ in the second step towards (17), where $(12 b)$ is quoted; while the contrary arrangement, density of $\chi_{s}(x)$ with potential 
$\chi_{s^{\prime}}(y)$, introduces the factor $m$ in the first step where $(10 b)$ is quoted, with an identical result.

For the volume potentials of magnetic type

and

$$
\frac{\rho}{4} \int_{\lambda}^{\infty} u_{\alpha}^{s}\left(1-u_{\alpha}\right)\left(\alpha x+\gamma^{\prime} y+\beta^{\prime} z\right) \frac{d \lambda}{\sqrt{J}}
$$

$$
\frac{\rho}{4} \int_{\lambda}^{\infty}\left(1-\|_{\alpha}\right)^{s}\left(\alpha x+\gamma^{\prime} y+\beta^{\prime} \approx \dot{z}\right) \frac{d \lambda}{\sqrt{ } \mathbf{J}},
$$

which we may call $\mathrm{U}_{s}^{\prime}(x), \mathrm{V}_{s}{ }^{\prime}(x)$ the composite terms in energy are respectively

and

$$
3 \rho^{2} \tau_{0} \mathrm{~L}_{0} /\left(2 s+2 s^{\prime}+3\right)\left(2 s+2 s^{\prime}+5\right)\left(2 s+2 s^{\prime}+7\right) .
$$

$$
\begin{aligned}
& \frac{3 \rho^{2} \tau_{0} \mathrm{~L}_{0}}{8} \cdot \frac{2.4 \ldots \overline{2 s+2 s^{\prime}}}{3.5 \ldots 2 s+2 s^{\prime}+3} \\
& \text { lieu of total charge in connexion with } \\
& \text { magnetic moment* defined for } \chi_{s}(x) \\
& m_{x}=\int x \rho_{s} d \tau=\frac{3 \rho \tau_{0}}{2(2 s+3)} ;
\end{aligned}
$$

for the function $\mathrm{U}_{s}^{\prime}(x)$ it is $\frac{3 \rho \tau_{0}}{\overline{2 s+3} \cdot \overline{2 s+5}}$. Thus an exact correspondence is given between the two types of potential.

$\S 6$. We now consider with reference to a potential

$$
\begin{aligned}
\mathrm{P}=k_{1} \mathrm{U}_{0} & +k_{2} \mathrm{U}_{1}+\ldots k_{n} \mathrm{U}_{n-1} \\
& =\frac{\rho}{4} \int_{\lambda}^{\infty}\left(1-u_{\alpha}\right)\left(k_{1}+k_{2} u_{\alpha}+k_{3} u_{\alpha}^{2}+\ldots k_{n} u_{\alpha}^{n-1}\right) \frac{d \lambda}{\sqrt{J}},
\end{aligned}
$$

the problem of determining the $k$ 's so as to make the energy $\mathrm{E}$ a minimum subject to the constancy of total charge $e, \mathrm{E}$ and $e$ being given by

$$
\left.\begin{array}{l}
\mathbf{E}=\frac{3 \rho^{2} \tau_{0} \phi_{0}}{2}\left[\frac{k_{1}^{2}}{1.3 .5}+\frac{2 k_{1} k_{\mathrm{g}}}{3.5 .7}+\frac{k_{2}^{2}+2 k_{1} k_{3}}{5.7 .9}+\ldots\right] \\
e=3 \rho \tau_{0}\left[\frac{k_{1}}{1.3}+3 \frac{k_{2}}{3.5}+\ldots\right]
\end{array}\right\}
$$

* In the case of E. (117) where these functions are used, it is, however, a question of total charge, and $e=3 \rho \tau_{\mathrm{o}} / 2$ for the conductor $(s=0), e=\rho \tau_{0}$ for the uniform volume distribution $(s=1)$. The energies for $\chi_{0}$ and for $\chi_{\mathrm{I}}$ are then respectively $e^{2} L_{0} / 36 \tau_{0}$ and $e^{2} L_{0} / 70 \tau_{0}$; thus if we seek the energy of convection currents due to surface charge instead of body charge, we must write in $F$. (117) $e^{2} / 36 \mathrm{~V}^{2}$ outside the square bracket. 
If a similar problem is stated for

$$
\mathrm{Q}=\frac{\rho}{4} \int_{\lambda}^{\infty}\left(1-u_{a}\right)\left(k_{1}+k_{2} u_{\alpha}+\ldots k_{n} u_{\alpha}^{n-1}\right)\left(\alpha x+\gamma^{\prime} y+\beta^{\prime} z\right) \frac{d \lambda}{\sqrt{J^{\prime}}}
$$

$\phi_{0}$ is replaced by $L_{0}$ and $e$ by $m_{x}$, and the coefficients open with $\frac{1}{3.5 .7}$ and $\frac{1}{3.5}$ instead of $\frac{1}{1.3 .5}$ and $\frac{1}{1.3}$. By writing $k=\frac{e \xi}{3 \rho \tau_{0}}$ for each subscript the minimum problem is presented with pure numbers, and we may generalize it by making the series of coefficients start at a different place. Thus using

$$
a_{p}=1 /(2 p-1)(2 p+1)(2 p+3) \text { and } b_{p}=1 /(2 p-1)(2 p+1) \text {, }
$$

it is required to find $\omega$ the minimum value of

where

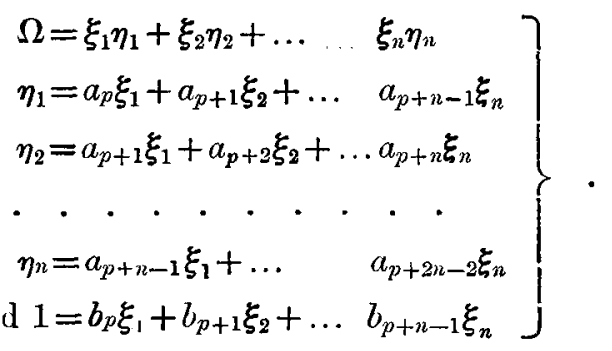

define the $\eta$ 's, and $1=b_{p} \xi_{1}+b_{p+1} \xi_{2}+\ldots b_{p+n-1} \xi_{n}$ is an equation of condition. From the point of view of potential theory, interest attaches to the value of the minimum, which proves to be

$$
\omega=\frac{2 p-1}{2} \cdot \frac{\overline{n+1} \overline{2 n+2 p-1}}{n \overline{2 n+2 p+1}} . .
$$

in the general case; and to the nature of the distribution which gives the minimum. For $p=1$ the case of (21),

$$
\omega=(n+1)(2 n+1) / 2 n(2 n+3) \text { and } \mathrm{E}=e^{2} \phi_{0} \omega / 6 \tau_{0} ;
$$

while the density for the potential $\mathrm{P}$ is

$$
\frac{e}{3 \tau_{0}}\left[\xi_{1}+\xi_{2}\left(-1+2 u_{a}\right)+\xi_{3}\left(-2 u_{a}+3 u_{a}^{2}\right) \ldots+\xi_{n}\left(-\overline{n-1} u_{a}^{n-2}+n u_{a}^{n-1}\right)\right]
$$

or

$$
\left.\begin{array}{l}
\frac{e}{3 \tau_{0}}\left[\xi_{1}-\xi_{2}+2\left(\xi_{2}-\xi_{3}\right) u_{a}+3\left(\xi_{3}-\xi_{4}\right) u_{a}^{2}+\ldots+n \xi_{n} u_{a}^{n-1}\right] \\
\text { or say } \\
\frac{e}{3 \tau_{0}}\left[x_{1}+w_{2} u_{a}+x_{3} u_{a}^{2}+\ldots+x_{n} u_{a}^{n-1}\right] .
\end{array}\right\}
$$


The conditions of no variation are

$$
\eta_{1}=\omega b_{1}, \eta_{2}=\omega b_{2}, \ldots \text { with } 1=b_{p} \xi_{1}+\ldots b_{p+n-1} \xi_{n} ;
$$

i. e.

$$
\left.\begin{array}{cc}
a_{p} \xi_{1}+\ldots & a_{p+n-1} \xi_{n}=\omega b_{p} \\
\cdot & \cdot \cdot \cdot \\
a_{p+n-1} \xi_{1}+\ldots a_{p+2 n-2} \xi_{n}=\omega b_{p+n-1} \\
b_{p} \xi_{1}+\ldots \quad b_{p+n-1} \xi_{n}=1
\end{array}\right\}
$$

Write the first as

$$
\begin{aligned}
\omega b_{p}= & \left(\xi_{1}-\xi_{2}\right) a_{p}+\left(\xi_{2}-\xi_{3}\right)\left(a_{p}+a_{p+1}\right) \\
& +\left(\xi_{3}-\xi_{4}\right)\left(a_{p}+a_{p+1}+a_{p+3}\right)+\ldots+\xi_{n}\left(a_{p}+\ldots a_{p+n-1}\right),
\end{aligned}
$$

and note that

$$
\begin{aligned}
a_{p}+a_{p+1} & +\ldots a_{p+n-1}=\frac{1}{4}\left(b_{p}-b_{p+n}\right) \\
& =\frac{1}{4}\left\{\frac{1}{\overline{2 p-1} \overline{2 p+1}}-\frac{1}{2 p+2 n-12 p+2 n+1}\right\} \\
& =\frac{1}{8}\left\{\frac{1}{2 p-1}-\frac{1}{2 p+1}-\frac{1}{2 p+2 n-1}+\frac{1}{2 p+2 n+1}\right\} \\
& =\frac{n}{4}\left\{\frac{1}{2 p-1} \overline{2 p+2 n-1}-\frac{1}{\overline{2 p+1} 2 p+2 n+1}\right\} .
\end{aligned}
$$

The first equation of (25) is then

$$
\begin{array}{r}
\frac{1}{2 p-1}\left[\frac{x_{1}}{2 p+1}+\frac{x_{2}}{2 p+3}+\ldots \frac{x_{n}}{2 p+2 n-1}\right]-\frac{1}{2 p+1}\left[\frac{x_{1}}{2 p+3}\right. \\
\left.+\ldots \frac{x_{n}}{2 p+2 n+1}\right]=4 \omega b_{p}=2 \omega\left(\frac{1}{2 p-1}-\frac{1}{2 p+1}\right)
\end{array}
$$

and others of this type are got by increasing $p$ by $1,2,3 \ldots$

Since $b_{p}+b_{p+1}+\ldots b_{p+n-1}=\frac{n}{2 p-1 \cdot 2 p+2 n-1}$, the equation of condition treated in this way becomes

$$
\frac{x_{1}}{2 p+1}+\frac{x_{2}}{2 p+3}+\ldots \frac{x_{n}}{2 p+2 n-1}=2 p-1 .
$$

The group of equations is then equivalent to

$$
\begin{aligned}
& \frac{x_{1}}{2 p+1}+\ldots \frac{x_{n}}{2 p+2 n-1}=2 \omega+\mathrm{C}(2 p-1), \\
& \frac{x_{1}}{2 p+3}+\ldots \frac{x_{n}}{2 p+2 n+1}=2 \omega+\mathrm{C}(2 p+1) \ldots,
\end{aligned}
$$


together with (26). The first with (26) gives $2 \omega+\mathrm{C}(2 p-1)$ $=2 p-1$, and the right hand of the $\left.\overline{q+1}\right|^{\text {th }}$ equation

$$
=2 \omega+\mathrm{C}(2 p+2 q-1)=2 p+2 q-1-\frac{4 q \omega}{2 p-1},
$$

which if the stated minimum (23) is correct

$$
=(2 p-1)\left[1-\frac{2 q}{n(2 p+2 n+1)}\right] \text {. }
$$

The group of equations is then

$$
\begin{aligned}
\frac{x_{1}}{2 p+2 q+1} & +\frac{x_{2}}{2 p+2 q+3}+\ldots \frac{x_{n}}{2 p+2 q+2 n-1} \\
& =\frac{2 p-1}{n(2 p+2 n+1)}[n(2 p+2 n+1)-2 q] .
\end{aligned}
$$

where $q$ has in succession the values $0,1,2 \ldots n$; if these are satisfied the original conditions are satisfied and $\omega$ has the value in $(23)$.

If $\mathrm{P}_{n}^{r}(\mu)$ is used for the $r^{\text {th }}$ differential coefficient of the zonal harmonic $\mathrm{P}_{n}(\mu)$, the solution of $(27)$ is comprised in the statement of the following as an identity, viz. :

$$
\begin{array}{r}
x_{1}+w_{2} \mu^{2} . \frac{2 p+1}{1}+x_{3} \mu^{4} \frac{\overline{2 p+1} \overline{\frac{2 p+3}{2 p}}}{1.3}+x_{4} \mu^{6} \frac{\overline{2 p+1} \overline{2 p+3} \overline{2 p+5}}{1.3 .5}+. . \\
\equiv \frac{(2 p-1) \mathrm{P}_{2 n+p}^{2+p}}{n(2 p+2 n+1) 1.3 \ldots 2 p-1} \cdot \quad \text { (28) }
\end{array}
$$

The numerical coefficients on the left hand are cleared by an integration $\int_{0}^{\mu} f d \mu$, followed by $p-1$ integrations

and the result is

$$
\mathrm{I} \cdot f=\int_{0}^{\mu} \mu f d \mu,
$$

$x_{1} \mu^{2 p-1}+x_{2} \mu^{2 p+1}+\ldots x_{n} \mu^{2 p+2 n-1} \equiv \frac{(2 p-1)}{n(2 p+2 n+1)} \mathrm{I}^{p-1} \cdot \mathrm{P}_{2 n+p}^{1+p} ;$

for

$$
p=1, x_{1} \mu+x_{2} \mu^{3}+\ldots=\mathrm{P}_{2 n+1}^{\prime \prime} / n(2 n+3), \quad . \quad .
$$

and for $p=2, x_{1} \mu^{3}+x_{2} \mu^{5}+\ldots=3\left(\mu \mathrm{P}_{2 n+2}^{\prime \prime}-\mathrm{P}_{2 n+2}^{\prime}\right) / n(2 n+5) .(29 c)$

The left-hand member of (27) is got from the left-hand member of (29) by multiplying by $\mu^{2 q+1}$ and integrating from 
$\mu=0$ to 1 , i.e. we require

$\left.\begin{array}{l}\int_{0}^{1} \mathrm{P}_{2 n+1}^{\prime \prime} \mu^{2 q+1} d \mu=n(2 n+3)-2 q, \text { for } p=1, \\ \int_{0}^{1}\left(\mu \mathrm{P}_{2 n+2}^{\prime \prime}-\mathrm{P}_{2 n+2}^{\prime}\right) \mu^{2 q+1} d \mu=n(2 n+5)-2 q, \text { for } p=2, \\ \text { and } \\ \int_{0}^{1} \mu^{2 q+1}\left(\mathrm{I}^{p-1} \cdot \mathrm{P}_{2 n+p}^{1+p}\right) d \mu=n(2 p+2 n+1)-2 q \\ \text { for the general case. }\end{array}\right\}$

Now

$$
\int_{0}^{1} \mathrm{P}_{2 n+1}^{\prime \prime} \mu^{2 q+1} d \mu=\mathrm{P}_{2 n+1}^{\prime}(1)-(2 q+1) \int_{0}^{1} \mu^{2 q} \mathrm{P}_{2 n+1}^{\prime} d \mu
$$

and

$$
\int_{0}^{1} \mu^{2 q} \mathrm{P}_{2 n+1}^{\prime} d \mu=\mathrm{P}_{2 n+1}(1)-2 q \int_{0}^{1} \mu^{2 q-1} \mathrm{P}_{2 n+1} d \mu
$$

the last term vanishes for $q=0,1,2 \ldots n$, but no further, and therefore

$\int_{0}^{1} \mathrm{P}_{2 n+1}^{\prime \prime} \mu^{2 q+1} d \mu=(n+1)(2 n+1)-(2 q+1)=n(2 n+3)-2 q$.

Again

$\int_{0}^{1}\left(\mu \mathrm{P}_{2 n+2}^{\prime \prime}-\mathrm{P}_{2 n+2}^{\prime}\right) \mu^{2 q+1} d \mu=\mathrm{P}_{2 n+2}^{\prime}(1)-(2 q+3) \int_{0}^{1} \mu^{2 q+1} \mathrm{P}_{2 n+2}^{\prime} d \mu$

while

$$
\int_{0}^{1} \mu^{2 q+1} \mathrm{P}_{2 n+2}^{\prime} d \mu=\mathrm{P}_{2 n+2}(1)-(2 q+1) \int_{0}^{1} \mu^{2 q} \mathrm{P}_{2 n+2} d \mu .
$$

The last integral vanishes for $q=0,1,2, \ldots n$, but no further, and therefore

$\int_{0}^{1}\left(\mu \mathrm{P}_{2 n+2}^{\prime \prime}-\mathrm{P}_{2 n+2}^{\prime}\right) \mu^{2 q+1} d \mu=(n+1)(2 n+3)-(2 q+3)=n(2 n+5)-2 q$.

The solution is therefore fully verified for the cases $p=1$ and 2, which belong to the two problems stated, the cases of potentials $P$ and $Q$. For the general case a mode of proof will be briefly indicated. Carry out the operation I by using $\mathrm{P}_{s+1}^{\prime}-\mu \mathrm{P}_{s}^{\prime}=(s+1) \mathrm{P}_{s}$ differentiated any number of times, hen in the result $\mathrm{I}^{p-1} \cdot \mathrm{P}_{2 n+p}^{p+1}$ yields

$$
\mathrm{P}_{2 n+2 p-1}^{\prime \prime}-(p-1)(2 n+2 p+1) \mathrm{P}_{2 n+2 v-2}^{\prime}+\text { a residue, }
$$


The residue can be expressed as a linear function of the P's of odd degree from $\mathrm{P}_{2 n+3}$ to $\mathrm{P}_{2 n+2 p+1}$, and the multiplication of these by $\mu^{2 q+1}$ and integration from 0 to 1 yields a zero result from $q=0$ to $q=n$. The integral on the left hand of $(30)$ is then

$$
\begin{gathered}
\int_{0}^{1} \mu^{2 q+1}\left[\mathrm{P}_{2 n+2 p-1}^{\prime \prime}-(p-1)(2 n+2 p+1) \mathrm{P}_{2 n+2 p-2}^{\prime}\right] d \mu=(n+p)(2 n+2 p-1) \\
-(2 q+1)-(p-1)(2 n+2 p+1)=n(2 n+2 p+1)-2 q \text { as required. }
\end{gathered}
$$

A comparison of $(29 b)$ with $(24)$ shows that the solution gives directly the density. Now a zonal harmonic has a point of inflexion between each maximum and minimum, therefore $\mu^{-1} \mathrm{P}_{2 n+1}^{\prime \prime}$ vanishes for $n-1$ values of $\mu^{2}$; hence the density vanishes for these values of $u_{a}$. The distribution is one in which there are $n$ concentric ellipsoidal shells of alternate positive and negative charges. When $n$ is indefinitely increased the charges on all but those for which $u_{u}$ is nearly $=1$ are indefinitely small, and the case becomes that of a condactor. A simple independent proof for the case of $n$ infinite shows the character of the limit. Since

$$
a_{p}+a_{p+1}+\ldots \text { ad inf. }=\frac{b_{p}}{4}
$$

the equation of condition may be written

$$
\frac{1}{4}=\xi_{1}\left(a_{p}+a_{p+1}+\ldots\right)+\xi_{2}\left(a_{p+1}+a_{p+2}+\ldots\right)+\ldots=\eta_{1}+\eta_{2}+\ldots
$$

i. e. it can be expressed in terms of the $\eta$ 's. But

$$
\delta \Omega=\xi_{1} \delta \eta_{1}+\ldots \text { subject to } 0=\delta \eta_{1}+\delta \eta_{2}+\ldots
$$

makes the $\xi$ 's all equal; and it is then easy to show that each $=2(2 p-1)$, and that $\omega=(2 p-1) / 2$. The potential $\mathrm{P}$ (case $p=1$ ) is then

$$
\frac{e}{b \tau_{0}} \int_{\lambda}^{\infty}\left(1-u_{\alpha}\right)\left(1+u_{\alpha}+u a_{\alpha}^{2}+\ldots\right) \frac{d \lambda}{\sqrt{\vec{J}}}=\frac{e}{b \tau_{0}} \int_{\lambda}^{\infty} \frac{d \lambda}{\sqrt{\vec{J}}},
$$

and the potential $Q$ (case $p=2$ ) is

$$
\begin{gathered}
\frac{m_{x}}{2 \tau_{\mathrm{o}}} \int_{\lambda}^{+\infty}\left(\alpha x+\gamma^{\prime} y+\beta^{\prime}\right)\left(1-u_{\alpha}\right)\left(1+u_{\alpha}+u^{2}+\ldots\right) \frac{d \lambda}{\sqrt{\widehat{J}}} \\
=\frac{m_{x}}{2 \tau_{\mathrm{o}}} \int_{\lambda}^{\infty} \frac{\left(\alpha x+\gamma^{\prime} y+\beta^{\prime} z\right)}{\sqrt{ } \bar{J}} .
\end{gathered}
$$

The higher derived functions which would correspond to higher values of $p$ in the minimum problem are not here constructed.

$\$ 7$. In conclusion we return to the general theorem of moments, give an independent proof, and then show how to 
deal with individual terms. We set out from the sphere, for which $\frac{1}{\tau_{\mathrm{o}}} \int x^{2 \nu_{1}} y^{2 v_{2} z v_{3}} d \tau$ $=\frac{1}{\tau_{0}} \int_{0}^{R} \int_{0}^{\pi} \int_{0}^{2 \pi} r^{2} \sin \theta d r d \theta d \phi(r \sin \theta \cos \phi)^{2 \nu}(r \sin \theta \sin \phi)^{2 \nu_{2}}(r \cos \theta)^{2 \nu_{3}}$ $=3 R^{2 \nu_{1}+2 \nu_{2}+2 \nu_{3}} \times \frac{1.3 \ldots \overline{2 \nu_{1}-1} \cdot 1 \cdot 3 \ldots 2 \nu_{2}-1.1 .3 \ldots \overline{2 \nu_{3}-1}}{1.3 \ldots 2 \nu_{1}+2 \nu_{2}+2 \nu_{3}+3} ;$

hence for an ellipsoid referred to principal axes

$$
a x^{2}+b y^{2}+c z^{2}=1, \text { with } \mathrm{A}=b c, \ldots
$$

$\frac{1}{\tau_{0}} \int x^{2 \nu_{1}} y^{2 \nu_{2}} z^{2 v_{3}} d \tau$

$=3\left(\frac{\mathrm{A}}{\Delta_{a}}\right)^{\nu_{1}}\left(\frac{\mathrm{B}}{\Delta_{a}}\right)^{\nu_{2}}\left(\frac{\mathrm{C}}{\Delta_{a}}\right)^{\nu_{3}} \times \frac{1.3 \ldots \overline{2 \nu_{1}-1} \cdot 1.3 \ldots \overline{2 \nu_{2}-1} \cdot 1 \cdot 3 \ldots \overline{2 \nu_{3}}-\overline{1}}{1.3 \ldots 2 v_{1}+2 v_{2}+2 v_{3}+3}$.

Therefore, if $2 s=2 v_{1}+2 v_{2}+2 v_{3}$,

$$
\begin{aligned}
& \frac{1}{\tau_{0}} \int(l x+m y+n z)^{2 s} d \tau=\Sigma \frac{\mid 2 s l^{2 \nu_{1}} m^{2 \nu_{2}} n^{2 \nu_{3}}}{\left|2 \nu_{3}\right| 2 \nu_{2} \mid 2 \nu_{3} \tau_{0}} \int x^{2 \nu_{1}} y^{2 \nu_{2} z^{2 \nu_{3}}} d \tau \\
& \left.=\frac{3}{(2 s+1)(2 s+3)} \Sigma \frac{\mid s}{\nu_{1} \mid \underline{\nu_{2}}} \mid \frac{\underline{\nu}_{3}}{\Delta_{a}}\right)^{\nu_{1}}\left(\frac{\mathrm{B} m^{2}}{\Delta_{a}}\right)^{\nu_{2}}\left(\begin{array}{c}
\mathrm{C} n^{2} \\
\Delta_{a}
\end{array}\right)^{\nu_{3}} \\
& =\frac{3}{(2 s+1)(2 s+3)}\left(\frac{\mathrm{A} l^{2}+\mathrm{B} m^{2}+\mathrm{C}^{2}}{\Delta_{a}}\right)^{s} \text {. }
\end{aligned}
$$

Now suppose $x y \approx$ transformed by a linear substitution to other axes $x_{1} y_{1} z_{1}$ which are not principal axes of the ellipsoid, and $l m n$ transformed by the inverse substitution to $l_{1} m_{1} n_{1}$ ( $c f$. Salmon, Higher Algebra, p. 102). The transformation makes $l x+m y+n z=l_{1} x_{1}+m_{1} y_{1}+n_{1} z_{1}$, leaves $\Delta_{a}$ unaltered, and gives $\mathrm{A}_{1} l_{1}^{2}+\ldots+2 \mathrm{~A}_{1}^{\prime} m_{1} n_{1} \ldots$ for $\mathrm{A} l^{2}+\mathrm{B} m^{2}+\mathrm{C} n^{2}$.

Hence dropping the subscripts we have the general form

$$
\frac{1}{\tau_{\mathrm{o}}} \int(l x+m y+n z)^{2 s} d \tau=\frac{3}{(2 s+1)(2 s+3)}\left(\frac{u_{\mathrm{A}}}{\Delta_{a}}\right)^{s} .
$$

In order to deal with specified products of inertia, a mode of writing the coefficients in the expansion of a ternary quadric is required, say

$\left.\begin{array}{rl}\quad\left\{u_{a}(x, y, z\}^{s}\right. & =\Sigma x^{\nu_{1}} y^{\nu_{2}} \tilde{z}^{\nu_{3}} \mathrm{~K}\left(\nu_{1}, \nu_{2}, \nu_{3} ; a\right) \\ 2 s & =\nu_{1}+\nu_{2}+\nu_{3}\end{array}\right\}$.

Phil. Mag. S. 6. Vol. 11. No. 64. April 1906. 2 Q 
Then comparing corresponding terms in the expansion of (11),

$$
\frac{1}{\tau_{0}} \int x^{\nu_{1}} y^{\nu_{2}} \tilde{z}_{3} d \tau=\frac{3\left|\nu_{1}\right| \nu_{2} \mid \nu_{3}}{(2 s+3)[2 s+1} \cdot \frac{\mathrm{K}\left(\nu_{1}, \nu_{2}, \nu_{3} ; A\right)}{\Delta_{u}^{s}} .
$$

The particular cases $s=1$ and 2 were given in $\approx$. p. 443 without proof. Making a similar application to the angular integral (3), the type of an individual term is

$$
\frac{1}{2 \pi} \int \frac{l \nu_{1} m v_{2} n \nu_{3} d \omega}{u_{p}\left(u_{\mathrm{A}}+\mu u_{p}\right)^{8+\frac{1}{2}}}=\frac{\left|\nu_{1}\right| \underline{\nu_{2}} \mid \underline{\nu_{3}}}{\mid 2 s . \Delta_{\alpha}^{s}} \int_{\mu}^{\infty} \frac{\mathrm{K}\left(\nu_{1}, \nu_{2}, \nu_{3} ; \alpha\right) d \mu}{\sqrt{\Delta(\mu)}} \text {. }
$$

The special cases $s=1$ and 2 were also given in $\mathbb{E}$. p. 445 and p. 459 .

The calculation of the coefficients $\mathrm{K}$ is facilitated by the use of sequence equations, obtained by differentiation of (31). Thus differentiation with regard to $x$ gives the first of

$$
\begin{aligned}
& \nu_{1} \mathrm{~K}\left(\nu_{1}, \nu_{2}, \nu_{3} ; a\right)=\left(\nu_{1}+\nu_{2}+\nu_{3}\right)\left[a \mathrm{~K}\left(\nu_{1}-2, \nu_{2}, \nu_{3}\right)+c^{\prime} \mathrm{K}\left(\nu_{1}-1 ; \nu_{2}-1, \nu_{3}\right)\right. \\
& \left.+b^{\prime} \mathrm{K}\left(\nu_{1}-1, \nu_{2}, \nu_{3}-1\right)\right] \\
& \nu_{2} \mathrm{~K}\left(\nu_{1}, \nu_{2}, \nu_{3} ; a\right)=\left(\nu_{1}+\nu_{2}+\nu_{3}\right)\left[e^{\prime} \mathrm{K}\left(v_{1}-1, \nu_{2}-1, v_{3}\right)+b \mathrm{~K}\left(\nu_{1}, v_{2}-2, \nu_{3}\right)\right. \\
& \left.+a^{\prime} \mathrm{K}\left(\nu_{1}, \nu_{2}-1, \nu_{3}-1\right)\right] \\
& \nu_{3} \mathrm{~K}\left(\nu_{1}, \nu_{2}, \nu_{3} ; a\right)=\left(\nu_{1}+\nu_{2}+\nu_{3}\right)\left[b^{\prime} \mathrm{K}\left(\nu_{1}-1, \nu_{2}, \nu_{3}-1\right)+a^{\prime} \mathrm{K}\left(\nu_{1}, \nu_{2}-1, \nu_{3}-1\right)\right. \\
& \left.+c \mathrm{~K}\left(\nu_{1}, \nu_{2}, \nu_{3}-2\right)\right] \text {. }
\end{aligned}
$$

If in $K\left(v_{1}, v_{2}, v_{3}\right)$ one of the indices is negative $\mathrm{K}=0$. There is a second group of relations of which the type is

$$
\begin{gathered}
\mathrm{A}\left(\nu_{1}+1\right) \mathrm{K}\left(\nu_{1}+1, \nu_{2}, \nu_{3} ; a\right)+\mathrm{C}^{\prime}\left(\nu_{2}+1\right) \mathrm{K}\left(\nu_{1}, \nu_{2}+1, \nu_{3} ; a\right) \\
+\mathrm{B}^{\prime}\left(\nu_{3}+1\right) \mathrm{K}\left(\nu_{1}, \nu_{2}, \nu_{3}+1 ; a\right) \\
=\left(\nu_{1}+\nu_{2}+\nu_{3}+1\right) \mathrm{K}\left(\nu_{1}-1, \nu_{2}, \nu_{3} ; a\right),
\end{gathered}
$$

got either by inverting the first group, or independently by the use of

$$
\left(\mathrm{A} \frac{d}{d x}+\mathrm{C}^{\prime} \frac{d}{d y}+\mathrm{B}^{\prime} \frac{d}{d z}\right) u_{a}^{s}=2 s \Delta_{a} \cdot x u_{a}^{s-1} .
$$

XLIX. The Absorption of the $\mathrm{y}$ Rays of Radioactive Substances.

By A. S. Eve, M.A., Lecturer in Mathematics, McGill University, Montreal *.

THE following investigations were made in order to 1 ascertain whether the $\gamma$ rays could be taken as an accurate measure of the total amount of radioactive matter present in a given substance. It was, therefore, necessary to ascertain whether the $\gamma$ rays of various substances were absorbed to an equal degree under the same conditions.

* Communicated by Prof. E. Rutherford, F.R.S. 